\title{
An Acquisition Algorithm with NCCFR for BOC Modulated Signals
}

\author{
Yongxin Feng, ${ }^{1}$ Fang Liu, ${ }^{2}$ Xudong Yao, ${ }^{2}$ and Xiaoyu Zhang ${ }^{2}$ \\ ${ }^{1}$ Communication and Network Institute, Shenyang Ligong University, Shenyang, Liaoning, China \\ ${ }^{2}$ School of Information Science and Engineering, Shenyang Ligong University, Shenyang, Liaoning, China \\ Correspondence should be addressed to Yongxin Feng; fengyongxin@263.net
}

Received 3 May 2017; Revised 24 June 2017; Accepted 2 July 2017; Published 3 August 2017

Academic Editor: Iickho Song

Copyright (c) 2017 Yongxin Feng et al. This is an open access article distributed under the Creative Commons Attribution License, which permits unrestricted use, distribution, and reproduction in any medium, provided the original work is properly cited.

\begin{abstract}
With the development of satellite navigation technology, BOC (Binary Offset Carrier) signals are proposed and applied in navigation system. However, in the advantages of enhancing the utilized rating of the band resource, some new problems are also emerging in the acquisition processing. On the basis of analyzing the limitations of the existing methods in suppressing side peaks, a NCCFR (New Cross-Correlation Function Reconstruction) algorithm is proposed, in which different modulation coefficients are used to construct correlation function with a shifter phase. The simulation results show that the new algorithm can suppress first side peaks and restrain other side peaks.
\end{abstract}

\section{Introduction}

With the continuous development of satellite communication technology, the limited frequency band resources become increasingly scarcity, so BOC modulation signal is proposed and used in satellite navigation to solve these problems. On the basis of traditional PSK signal, BOC signal owns superior spectrum splitting characteristics, which can solve the frequency resource shortage problem; meanwhile it has better acquisition precision. However, the BOC signal also brings some problems, such as the acquisition ambiguity problem caused by multipeak characteristics [1].

Many effective acquisition methods [2,3] have been proposed to solve some acquisition problems, and some tracking technology [4] is proposed for BOC signals. In addition, some methods are proposed to remove side peaks, such as the BPSK-like method [5], ASPeCT method, and RQCC (Remove Quadratic BOC Cross-Correlation) reconstruction algorithm. In BPSK-like method, the frequency domain of BOC signals is regarded as the two BPSK signals. On the basis of filtering and frequency transform to the side-lobe of BOC signal, the acquisition processing is accomplished by using conventional BPSK acquisition method $[6,7]$. In ASPeCT method, the cross-correlation function of the signal and PRN code is applied, which is constructed by pseudorandom code to restrain the side peaks [8]. Using appropriate phase shift of the cross-correlation function and nonrelated accumulation about the shifted correlation function, the filtered method can enhance the main peak and restrain the side peaks [9]. In RQCC method, the new QBOC (Quadratic Binary Offset Carrier) auxiliary signal is reconstructed by using the characteristics of same autocorrelation function peak width between the auxiliary signal and BOC signals to solve the problem that the side peaks restrained weakly, which is caused by different correlation function peak width between the PRN auxiliary signal and BOC signals [10]. On the basis of the shifted correlation function phase, by introducing QBOC auxiliary signal and adding the shifted results to eliminate the side peaks, these methods can solve some multipeak problems, but they cannot restrain the multipeak problem in different modulation coefficient and meanwhile ensure main peak sharply.

Therefore, considering the acquisition ambiguity problems and the shortage of the existing methods, a NCCFR (New Cross-Correlation Function Reconstruction) acquisition algorithm is proposed. Using different modulation coefficient to make a different fixed phase shifter correlation function, the algorithm can efficiently restrain the first side peaks and improve the main peak. 


\section{NCCFR Acquisition Algorithm}

Firstly, an QBOC auxiliary signal $[11,12]$ can be got by $\pi / 2$ shifting the phase of local BOC signals, and then the BOC signal and the QBOC signal are defined in formulas (1) and (2). Then the autocorrelation function of receiving signal $R_{X}(\tau)$ will be got through correlation processing, and the cross-correlation function $R_{X / Q}(\tau)$ will be got by correlation processing between the auxiliary signal and received signal, which is shown in formulas (3) and (4), respectively, where $C(n)$ is the baseband code, $\mathrm{SN}(n)$ is the square wave, and $\operatorname{tri} i_{x}(\tau)$ is the correlation peak whose position is $x$.

$$
\begin{aligned}
& \operatorname{BOC}(m, n)=C(n) \mathrm{SN}(2 \pi \omega n+\theta) \\
& \operatorname{QBOC}(m, n)=C(n) \mathrm{SN}\left(2 \pi \omega n+\frac{\pi}{2}+\theta\right) \\
& R_{X}(\tau)=\sum_{i=1}^{n-1}\left(\left[(-1)^{i} \operatorname{tri}_{-i / n}(\tau)+(-1)^{i} \operatorname{tri}_{i / n}(\tau)\right]\right. \\
& \left.\quad \times \frac{n-i}{n}\right)+\operatorname{tri}_{0}(\tau)=\sum_{i=-(n-1)}^{n-1}(-1)^{|i|} \operatorname{tri}_{-i / n}(\tau) \frac{n-|i|}{n} \\
& R_{X / Q}(\tau)=\sum_{i=1}^{n}\left(\left[(-1)^{i} \operatorname{tri}_{-(2 i-1) / 2 n}(\tau)\right.\right. \\
& \left.\left.\quad+(-1)^{i+1} \operatorname{tri}_{(2 i-1) / 2 n}(\tau)\right] \times \frac{2 n-(2 i-1)}{2 n}\right) .
\end{aligned}
$$

Due to the difference of correlation peak phase between $R_{X / Q}(\tau)$ and $R_{X}(\tau)$, the main peak and some side peaks, which is similar to $R_{X}(\tau)$, can be reconstructed. Therefore, the nonconstant phase shift of $R_{X / Q}(\tau)$ is taken both early and late, $\left((n-1) T_{c}\right) / 2 n$, where $n$ is the modulation coefficient, $T_{c}$ is the period of one chip, and $\tau$ is the chip delay. As formulas (5) and (6) show, the superfluous side peaks will be eliminated as much as possible by taking addition and subtraction, which is shown in formula (7) as follows:

$$
\begin{aligned}
& R_{X / Q}\left(\tau-\frac{(n-1) T_{c}}{2 n}\right)=\sum_{i=1}^{n}\left(\left[(-1)^{i} \operatorname{tri}_{-(2 i+n-2) / 2 n}(\tau)\right.\right. \\
& \left.\left.+(-1)^{i+1} \operatorname{tri}_{(2 i-n) / 2 n}(\tau)\right] \times \frac{2 n-(2 i-1)}{2 n}\right) \\
& R_{X / Q}\left(\tau+\frac{(n-1) T_{c}}{2 n}\right)=\sum_{i=1}^{n}\left(\left[(-1)^{i} \operatorname{tri}_{-(2 i-n) / 2 n}(\tau)\right.\right. \\
& \left.\left.+(-1)^{i+1} \operatorname{tri}_{(2 i+n-2) / 2 n}(\tau)\right] \times \frac{2 n-(2 i-1)}{2 n}\right) \\
& M=\left|R_{X / Q}\left(\tau-\frac{(n-1) T_{c}}{2 n}\right)\right|+\mid R_{X / Q}(\tau \\
& \left.+\frac{(n-1) T_{c}}{2 n}\right)|-| R_{X / Q}\left(\tau-\frac{(n-1) T_{c}}{2 n}\right)
\end{aligned}
$$

$$
\begin{aligned}
& +R_{X / Q}\left(\tau+\frac{(n-1) T_{c}}{2 n}\right) \mid=\frac{1}{n}\left(\operatorname{tri}_{-1 / 2}(\tau)\right. \\
& \left.+\operatorname{tri}_{1 / 2}(\tau)\right)+\sum_{i=-n / 2+1}^{n / 2-1} \operatorname{tri}_{-i / n}(\tau)\left(\frac{n+1}{n}-\frac{|2 i|}{n}\right),
\end{aligned}
$$

where $\operatorname{tri}_{ \pm i / n}(\tau)$ represents the correlation peak in location $\pm i / n$.

There are many correlation peaks similar to $R_{X}(\tau)$ in formula (7), especially the main peak and the correlation peak before $n / 2$. Therefore, the reconstructed signal can improve main peak and restrain the side peaks of the correlation function and then can obtain formula (8) as follows:

$$
\begin{aligned}
R_{X}(\tau) & +M \\
= & \sum_{i=-(n-1)}^{n-1}(-1)^{|i|} \operatorname{tri}_{-i / n}(\tau) \frac{n-|i|}{n} \\
& +\frac{1}{n}\left(\operatorname{tri}_{-1 / 2}(\tau)+\operatorname{tri}_{1 / 2}(\tau)\right) \\
& +\sum_{i=-n / 2+1}^{n / 2-1} \operatorname{tri}_{-i / n}(\tau)\left(\frac{n+1}{n}-\frac{|2 i|}{n}\right) \\
= & \sum_{i=-n / 2+1}^{n / 2-1} \operatorname{tri}_{-i / n}(\tau)\left[(-1)^{|i|} \frac{n-|i|}{n}+\frac{n+1}{n}-\frac{|2 i|}{n}\right] \\
& +\left(\frac{1}{n}+\frac{1}{2}\right)\left(\operatorname{tri}_{-1 / 2}(\tau)+\operatorname{tri}_{1 / 2}(\tau)\right) \\
& +\sum_{i=n / 2+1}^{n-1}(-1)^{|i|} \frac{n-|i|}{n}\left(\operatorname{tri}_{-i / n}(\tau)+\operatorname{tri} i_{i / n}(\tau)\right) .
\end{aligned}
$$

According to formula (8), when $i=0, \operatorname{tri}_{0}(\tau)$ represents the main correlation peak of the signal, and the main peak will promote $(n+1) / n$. Not only will the signal synchronization acquisition rate of receiver be greatly improved, but also the acquisition ambiguity of the signal will be reduced effectively, when $i=1$, and the results of both $\operatorname{tri}_{1 / n}(\tau)$ and $\operatorname{tri} i_{-1 / n}(\tau)$ are 0 , indicating that the first side peak is eliminated. In the odd condition, the absolute value of $(-1)^{|i|}((n-|i|) / n)+$ $(n+1) / n-|2 i| / n$ is less than $(-1)^{|i|}((n-|i|) / n)(-1)^{|i|}((n-$ $|i|) / n)$. Therefore, the odd correlation peak before $n / 2$ after the reconstruction can be restrained, and the even correlation peak can be enhanced.

Because there are no high rate subcarrier signals mixed, making the peak width $R_{X / P}(\tau)$ of both auxiliary signal PRN and the received BOC signals wider than $R_{X}(\tau)$, which can restrain the side peaks in some extent, auxiliary signal PRN can be introduced to restrain the even peak and the correlation peak after $n / 2$. The cross-correlation function is shown in formula (9).

$$
R_{X / P}(\tau)=\sum_{i=1}^{n / 2}\left(\left[-\operatorname{tri} i_{-(2 i-1) / n}(\tau)+\operatorname{tri}_{(2 i-1) / n}(\tau)\right] \times \frac{1}{n}\right) .
$$




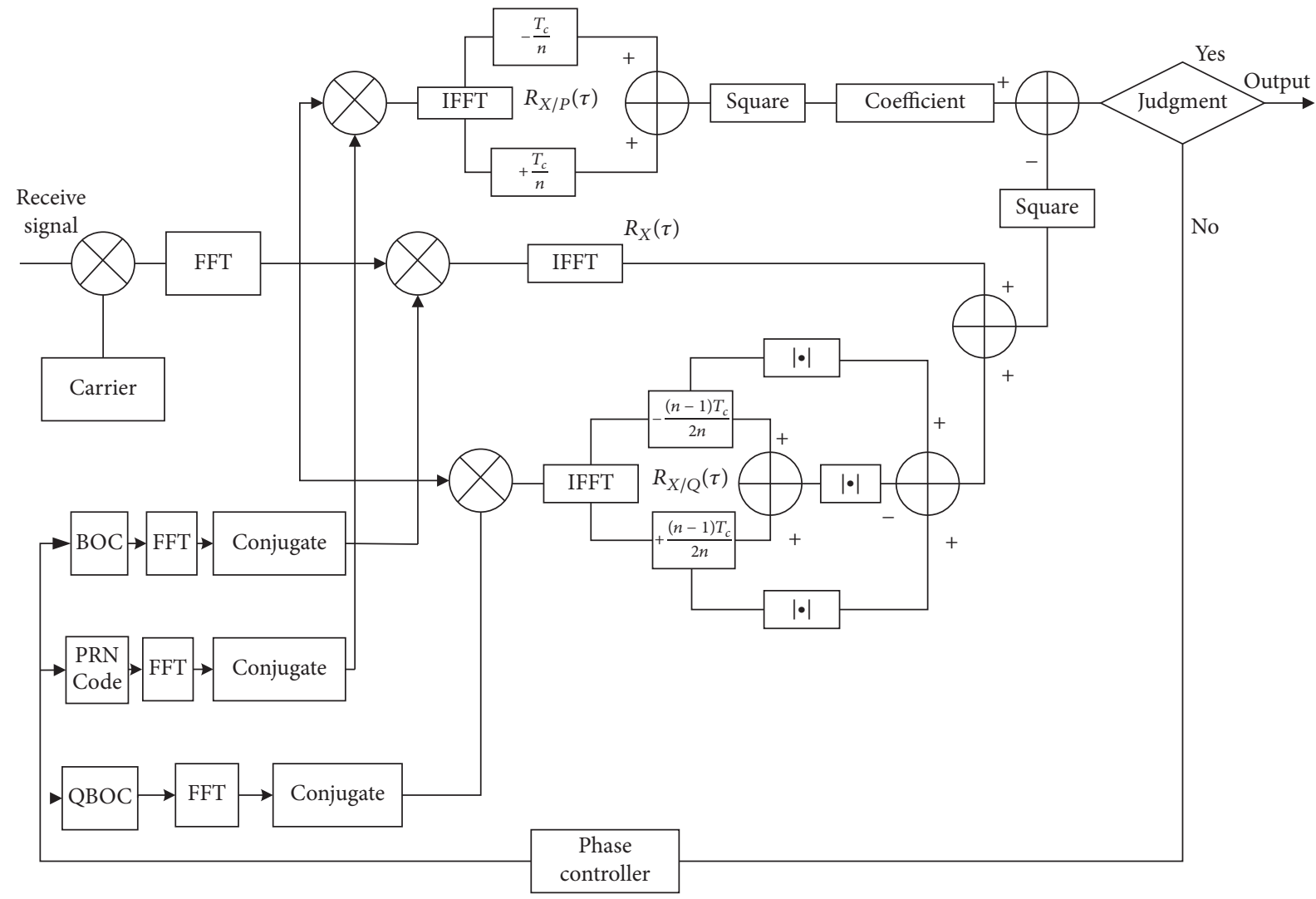

FIgURE 1: The principle of NCCFR.

In order to get the reconstructed signal which only consists of the even peak, by phase shift of the cross-correlation function (CCF), we can get formula (10).

$$
\begin{aligned}
& \left|R_{X / P}\left(\tau+\frac{T_{c}}{n}\right)+R_{X / P}\left(\tau-\frac{T_{c}}{n}\right)\right| \\
& =\sum_{i=1}^{n / 2-1}\left(\left[\operatorname{tri}_{-2 i / n}(\tau)+\operatorname{tri}_{2 i / n}(\tau)\right] \times \frac{2}{n}\right)+\frac{1}{n} \\
& \quad \times\left(\operatorname{tri}_{1}(\tau)+\operatorname{tri}_{-1}(\tau)\right) .
\end{aligned}
$$

According to the characteristics that there is only even peak appearance in formulas (8) and (10), the constant coefficient, coef, can adjust the correlation peak value in formula (10); moreover the acquisition results of the correlation side peaks will be restrained. Therefore the final formula of NCCFR can be expressed as follows by the similar processing as formula (11) between formulas (8) and (10):

$$
\begin{aligned}
& {\left[R_{X}(\tau)+\left(\left|R_{X / Q}\left(\tau-\frac{(n-1) T_{c}}{2 n}\right)\right|\right.\right.} \\
& +\left|R_{X / Q}\left(\tau+\frac{(n-1) T_{c}}{2 n}\right)\right| \\
& \quad-\mid R_{X / Q}\left(\tau-\frac{(n-1) T_{c}}{2 n}\right)
\end{aligned}
$$

$$
\begin{aligned}
& \left.\left.+R_{X / Q}\left(\tau+\frac{(n-1) T_{c}}{2 n}\right) \mid\right)\right]^{2}-\left[\operatorname{coef} \times \mid R_{X / P}(\tau\right. \\
& \left.\left.-\frac{T_{c}}{n}\right)+R_{X / P}\left(\tau-\frac{T_{c}}{n}\right) \mid\right]^{2} .
\end{aligned}
$$

Combined with the principle of NCCFR, the principle based on FFT (Fast Fourier Transform) is shown in Figure 1.

\section{Algorithm Simulation and Test}

3.1. Comparison Analysis of the Reconstruction Algorithm. In order to verify the performance of the new algorithm in restraining side peaks, according to the design model of Figure 1, reconstruction comparison analysis of different algorithm is fulfilled based on several typical BOC signals. The simulation parameters are as follows:

(1) $\operatorname{BOC}(2,2)$ signal, pseudorandom code rate is $2 \times$ $1.023 e^{6}$, and subcarrier rate is $2 \times 1.023 e^{6}$;

(2) $\operatorname{BOC}(8,4)$ signal, pseudorandom code rate is $4 \times$ $1.023 e^{6}$, and subcarrier rate is $8 \times 1.023 e^{6}$;

(3) $\operatorname{BOC}(6,1)$ signal, pseudorandom code rate is $1 \times$ $1.023 e^{6}$, and subcarrier rate is $6 \times 1.023 e^{6}$. 


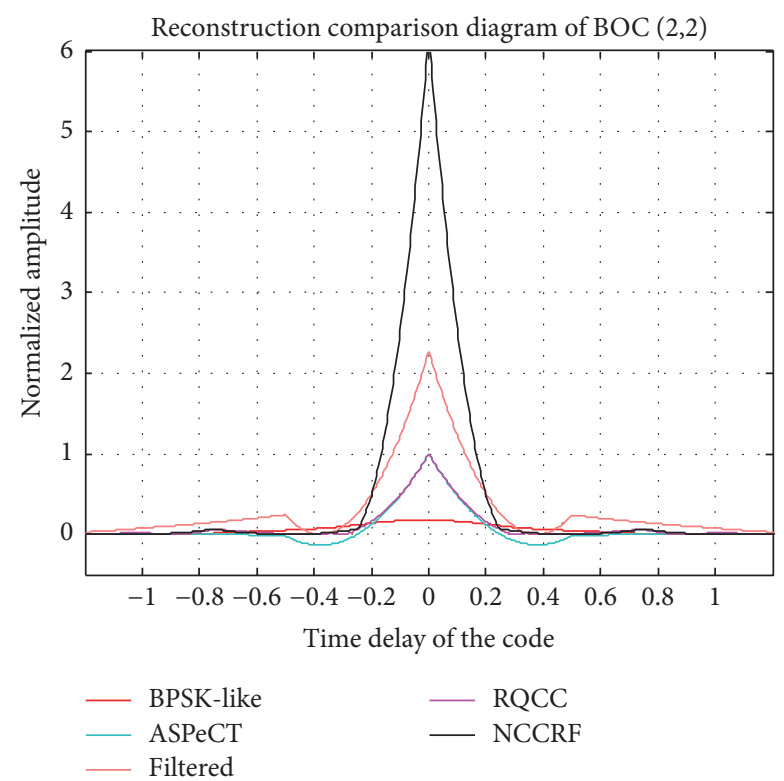

FIGURE 2: The reconstruction comparison of $\operatorname{BOC}(2,2)$.

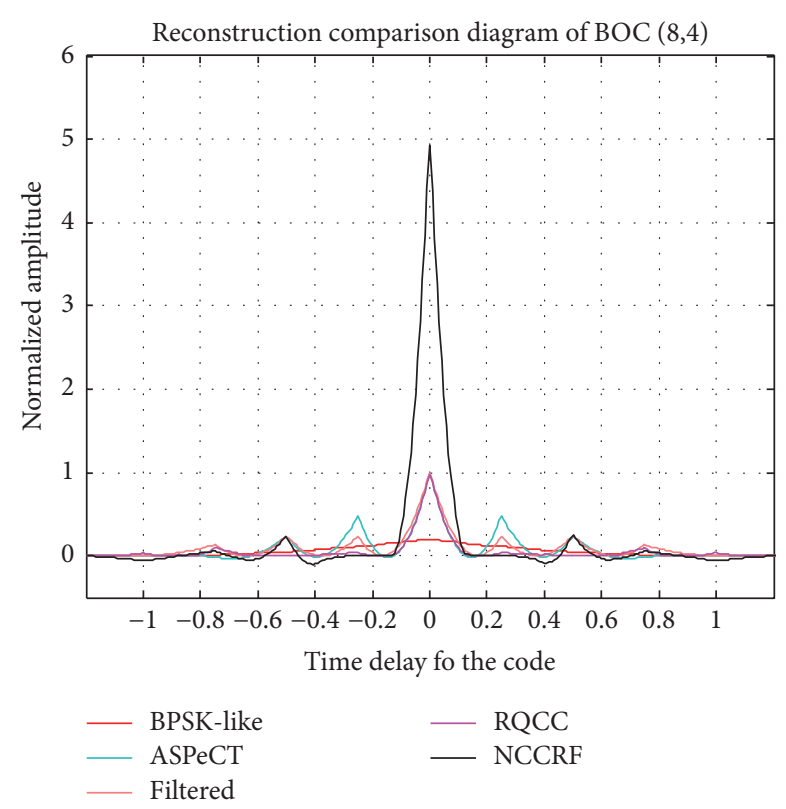

FIgURE 3: The reconstruction comparison of $\operatorname{BOC}(8,4)$.

The carrier rate and sampling rate are $30 \times 1.023 e^{6}$ and $480 \times 1.023 e^{6}$, respectively; the simulation results are shown in Figures 2-4.

They show the side peaks restrained performance promoted in NCCFR correlation reconstruction for $\operatorname{BOC}(2,2)$ signal from Figure 2, in which the side peaks have almost been eliminated. It can be seen from Figure 3 that the first side peak has been eliminated in NCCFR for BOC $(8,4)$ signal, although new side peaks are produced, whose ratio is smaller than the promotion of the main peak. From Figure 4 it can be seen that the first side peak has been eliminated in NCCFR for $\operatorname{BOC}(6,1)$ signal, but it also produces new larger side

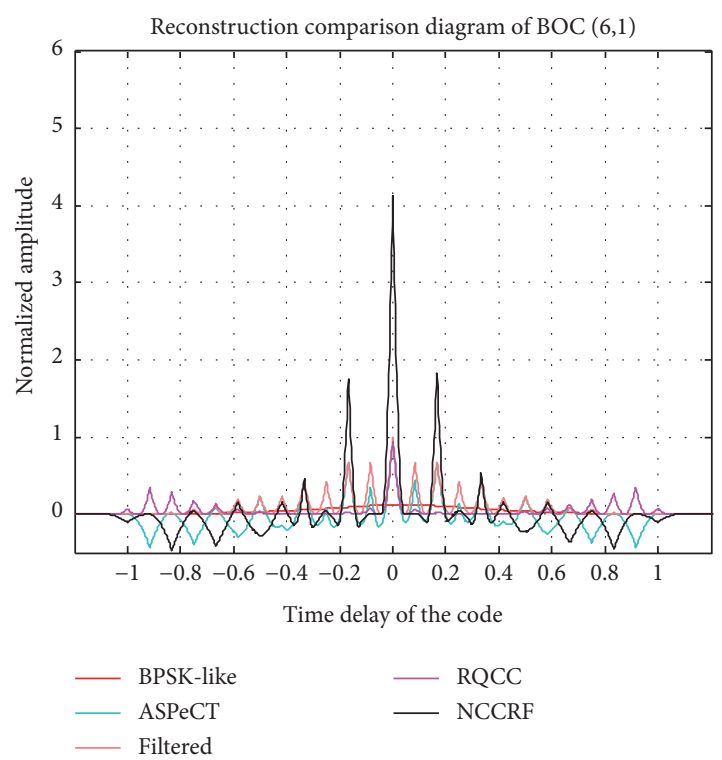

FIGURE 4: The reconstruction comparison of $\operatorname{BOC}(6,1)$.

peaks. In conclusion, NCCFR has very good performance in side peaks restrained for low order modulation coefficient of BOC signals and weaker peaks restrained performance for high order modulation coefficient.

3.2. Comparison Analysis of the Correlation Value. Moreover, in order to validate the adaptability of the algorithm in noisy environment, comparison analysis should be done. And MPMR is defined, which is the ratio between the main peak and the mean peak, MSPR is defined, which is the ratio between the main peak and the side peak, and MFSPR is defined, which is the ration between the main peak and the first side peak, where the first side peaks represent the second high degree of the peak [13].

First of all, the main peak enhanced performance is analyzed from MPMR [14-16]. The simulation results are shown in Figures 5-7.

It shows that the main peak enhanced performance promoted in NCCFR correlation reconstruction for both BOC $(2,2)$ and the $\operatorname{BOC}(8,4)$ from Figures 5 and 6 , combined with the formula analysis, and NCCFR algorithm enhance $(n+1) / n$ of the main peak, so main peak enhanced performance will be shown for low order BOC signal. But it is weaker than RQCC in high order modulation coefficient that is because the algorithm will produce new correlation side peaks by using ASPeCT acquisition algorithm which will restrain the correlation peak after $n / 2$.

Secondly, the correlation peak enhanced performance also contains the restrain performance of the side peaks. Therefore, acquisition performance can be analyzed from MSPR, which is shown in Figures 8-10.

The results show that all MSPR for different BOC signals in NCCFR is the biggest, indicating that the algorithm has good performance in restraining the side peaks and enhancing the main peak for different order modulation coefficient BOC signals; combined with the formula analysis, 


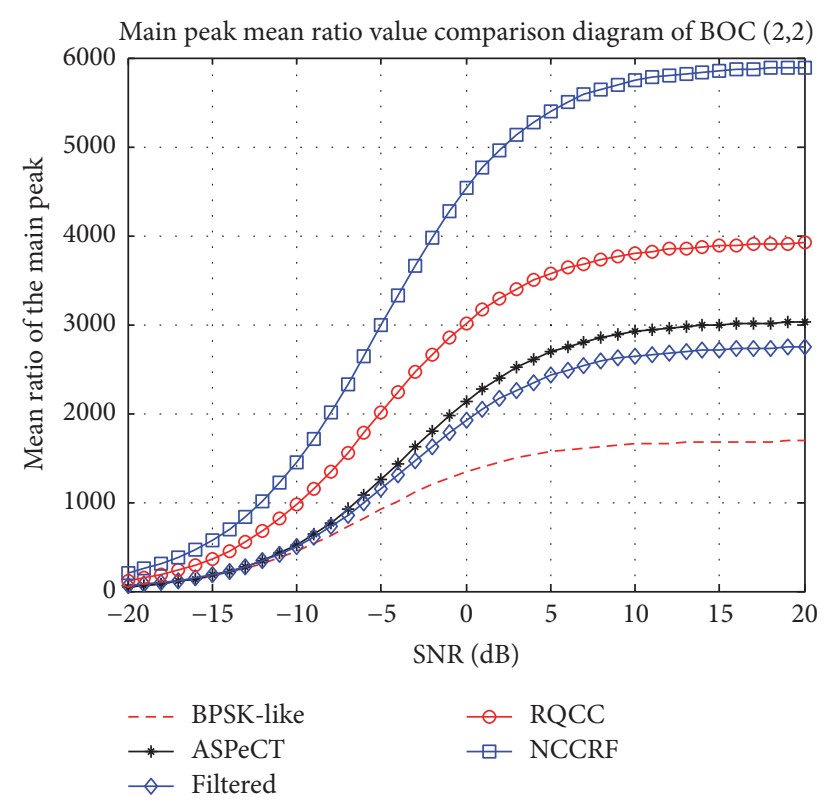

FIgURE 5: The MPMR comparison of $\operatorname{BOC}(2,2)$.

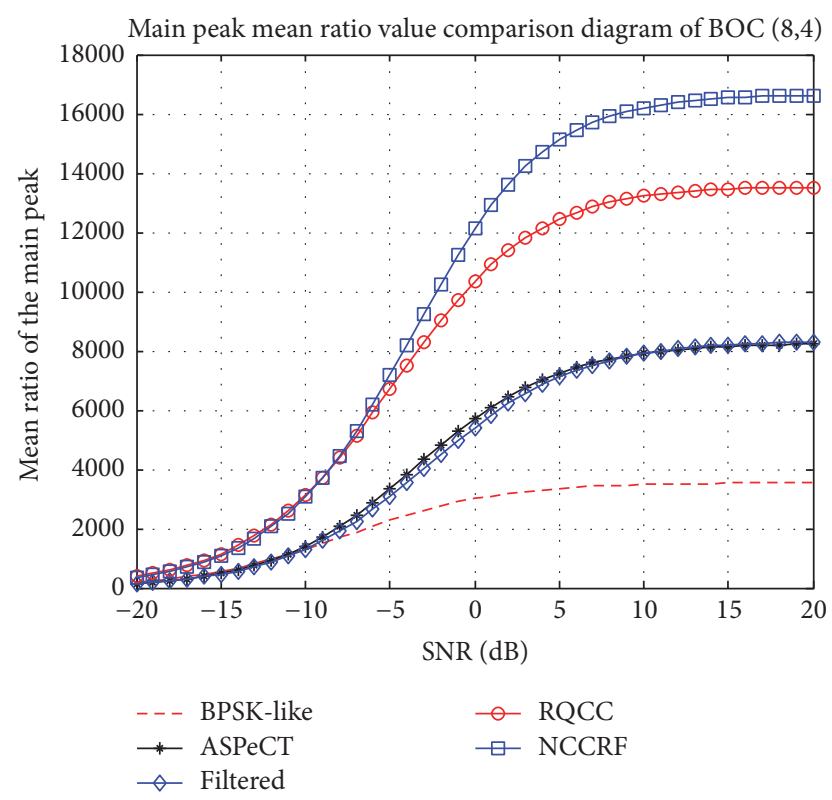

FIgURE 6: The MPMR comparison of $\operatorname{BOC}(8,4)$.

the new algorithm has better side peaks restrained ability; that is, NCCFR has the best enhanced performance on MSPR.

Finally, the first side peaks restrain performance is analyzed for MFSPR in different conditions of SNR. Considering that there are no other side peaks in NCCFR reconstruction for low order BOC $(2,2)$, the MFSPR is analyzed for both $\operatorname{BOC}(8,4)$ and $\operatorname{BOC}(6,1)$, which is shown in Figures 11 and 12.

The results show that MFSPR for BOC $(8,4)$ in NCCFR is the biggest, indicating that the algorithm has good performance in restraining the first side peaks. At the same

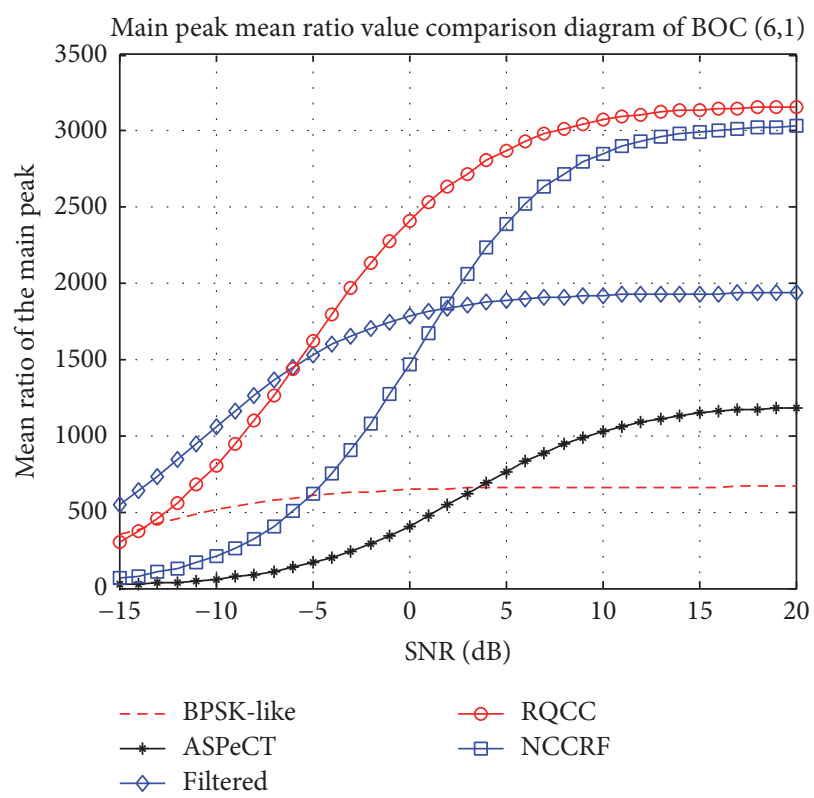

FIgURE 7: The MPMR comparison of $\operatorname{BOC}(6,1)$.

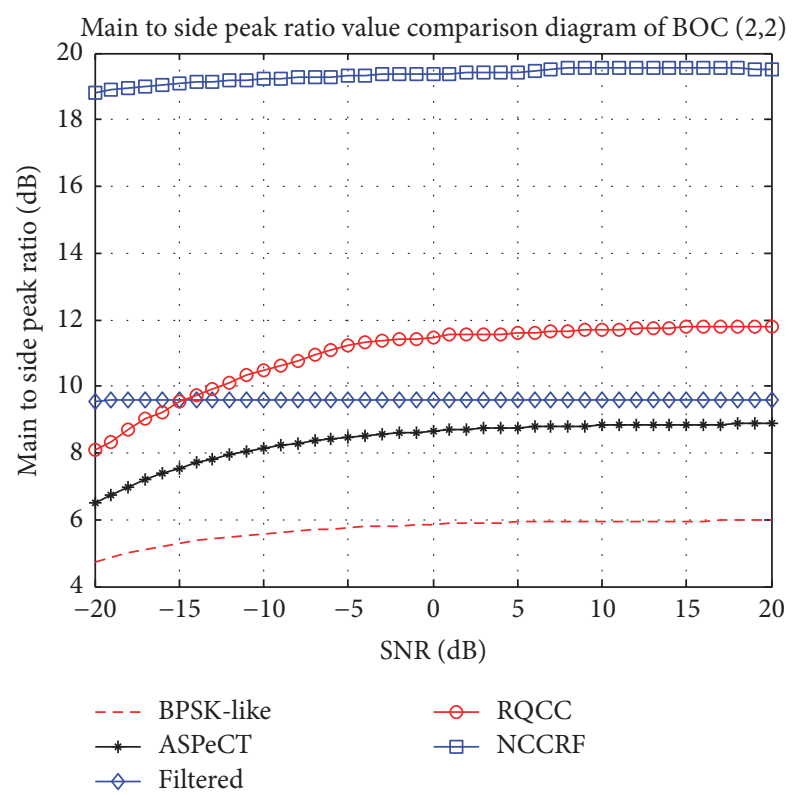

Figure 8: The MSPR comparison of $\operatorname{BOC}(2,2)$.

time, performance in restraining the first side peaks of NCCFR is weaker than RQCC for BOC $(6,1)$, but in general the new algorithm has a better first side peaks restrained performance.

3.3. Comparison Analysis of the Detection Probability. Signal acquisition probability is another important capability for signal acquisition, including the detection probability and false alarm probability $[17,18]$. Noise will exist to the received signal during the transmission, which probability usually obeys the noncentral chi square distribution; however pure 


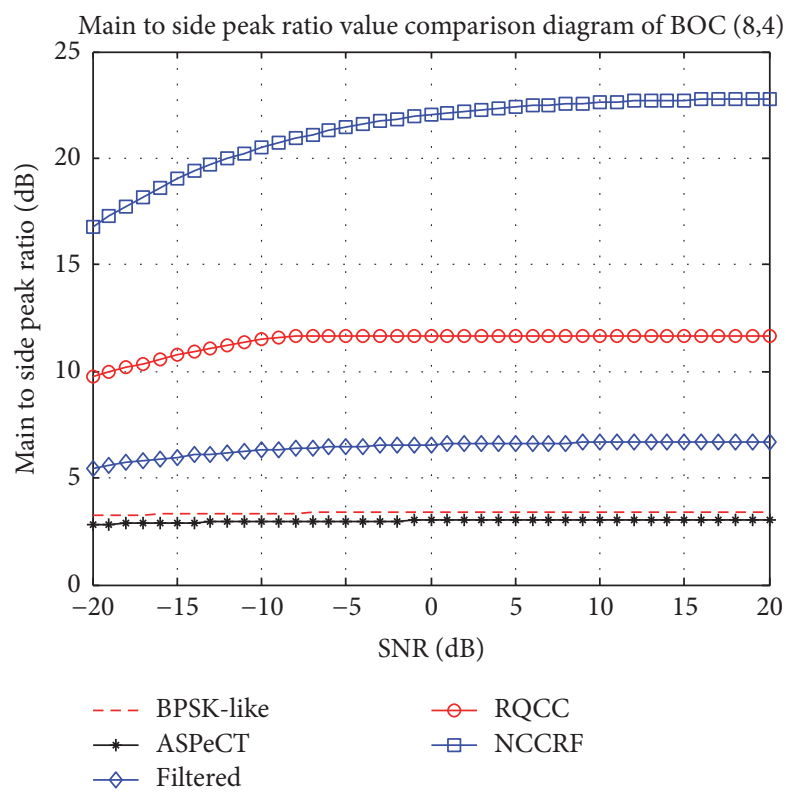

FIGURE 9: The MSPR comparison of $\operatorname{BOC}(8,4)$.

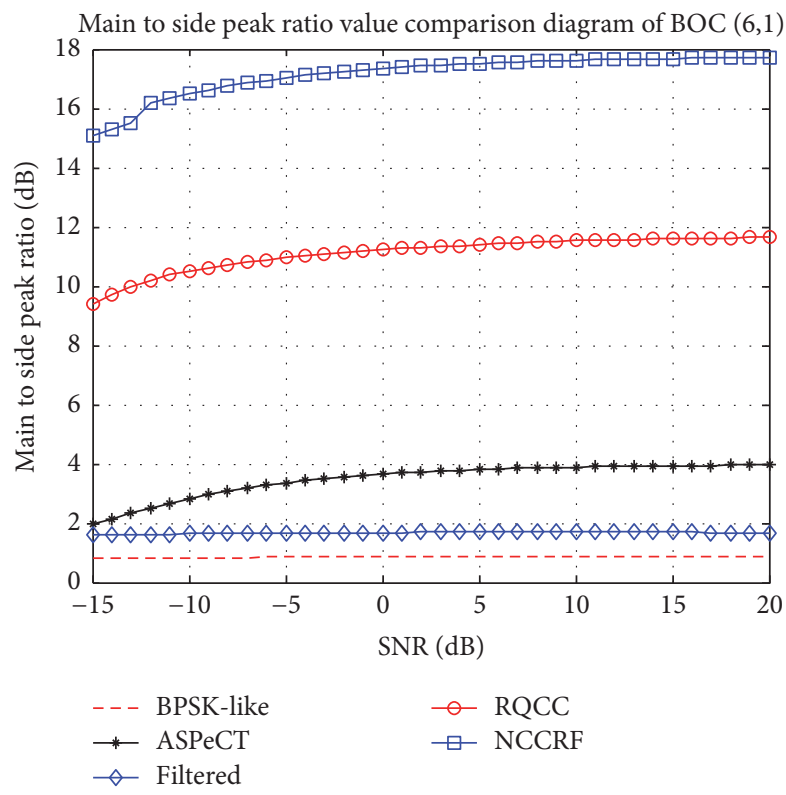

Figure 10: The MSPR comparison of $\operatorname{BOC}(6,1)$.

noise or nonsynchronized signal usually obeys the noncentral chi square distribution [19]. Furthermore, in order to validate the acquisition performance of NCCFR, setting the parameter of false alarm probability, pfa $=0.01$, which is a constant value, comparison analysis has been done from the correlation peak detection probability according to several typical signals in different SNR conditions, which mainly focuses on the main peak detection probability (MPDR), the side peaks detection probability (SPDR), and the first side peaks detection probability (FSPDR).

Firstly, the MPDR has been analyzed in different SNR conditions, which are shown in Figures 13-15.

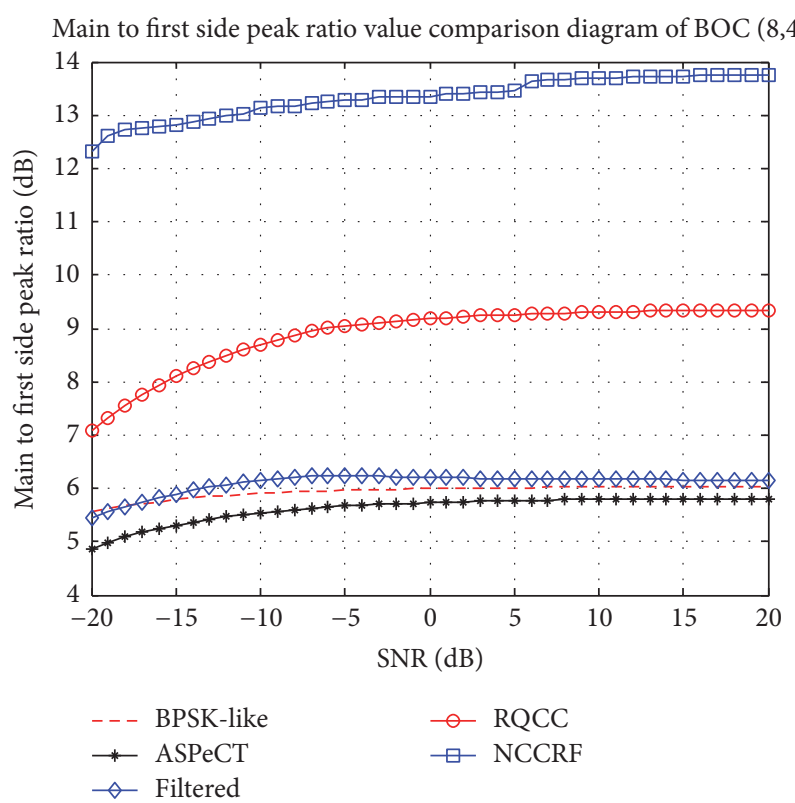

FIgURE 11: The MFSPR comparison of $\operatorname{BOC}(8,4)$.

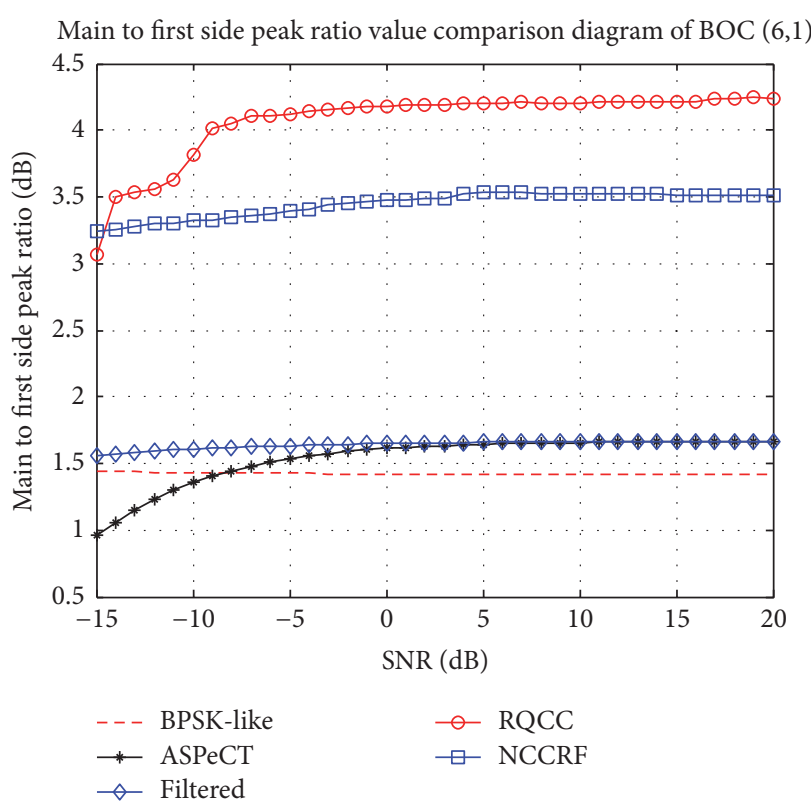

FIgURE 12: The MFSPR comparison of BOC $(6,1)$.

The results show that MPDR performance for both $(2,2)$ and BOC $(8,4)$ in NCCFR is better than others and MPDR performance of NCCFR is weaker than RQCC for BOC $(6,1)$, but in general the new algorithm has a better MPDR performance.

Secondly, according to the ambiguity problem of the side peaks, the SPDR has been analyzed in different SNR conditions, which are shown in Figures 16-18.

The results show that SPDR for NCCFR in different SNR conditions is the smallest one, indicating that the algorithm 


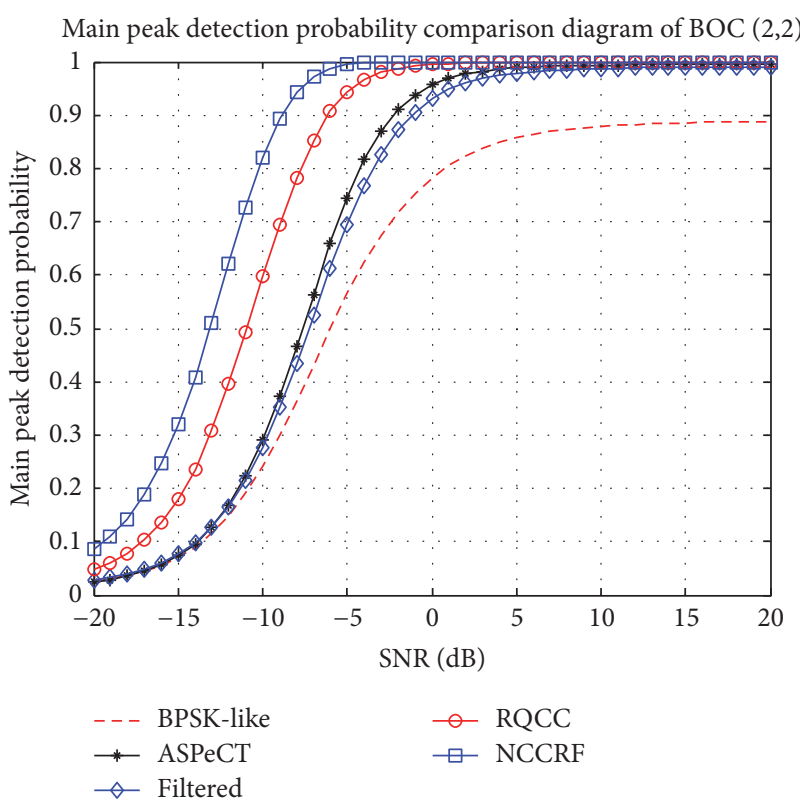

FIgURE 13: The MPDR comparison of $\operatorname{BOC}(2,2)$.

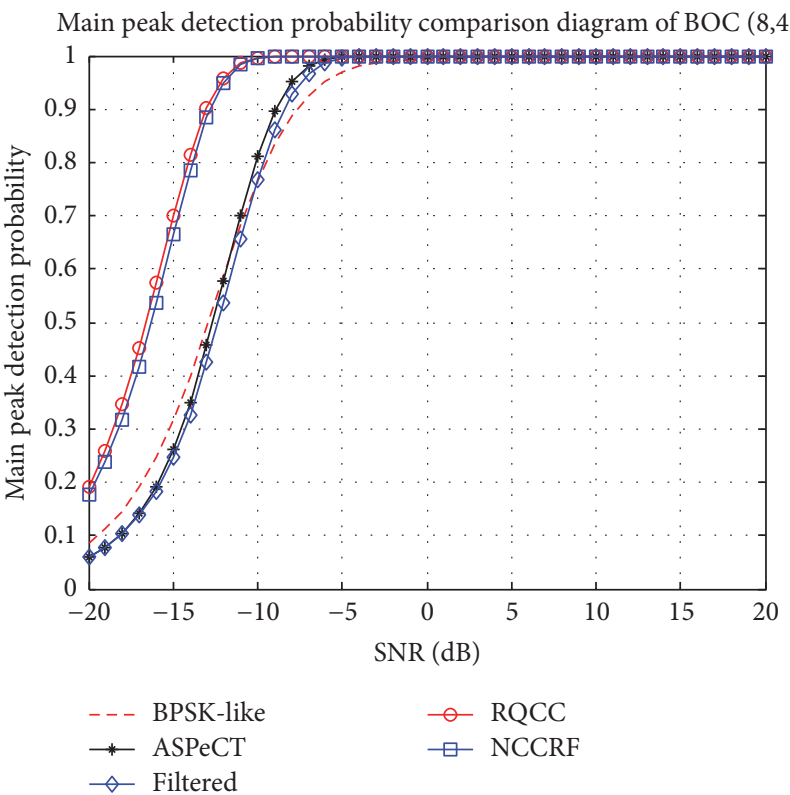

FIgURE 14: The MPDR comparison of $\operatorname{BOC}(8,4)$.

has good performance in restraining the side peaks for BOC signals.

Finally, according to the problem of producing new side peaks in reconstruction algorithm, the acquisition performance has been analyzed in different SNR conditions focusing on FSPDR, which are shown in Figures 19-20.

The results show that algorithm has good performance in restraining the first side peaks for BOC $(8,4)$ signal. From Figure 20 it can be seen that the performance in restraining the first side peaks of NCCFR is better than others when the SNR is low, but weaker than RQCC when the SNR is

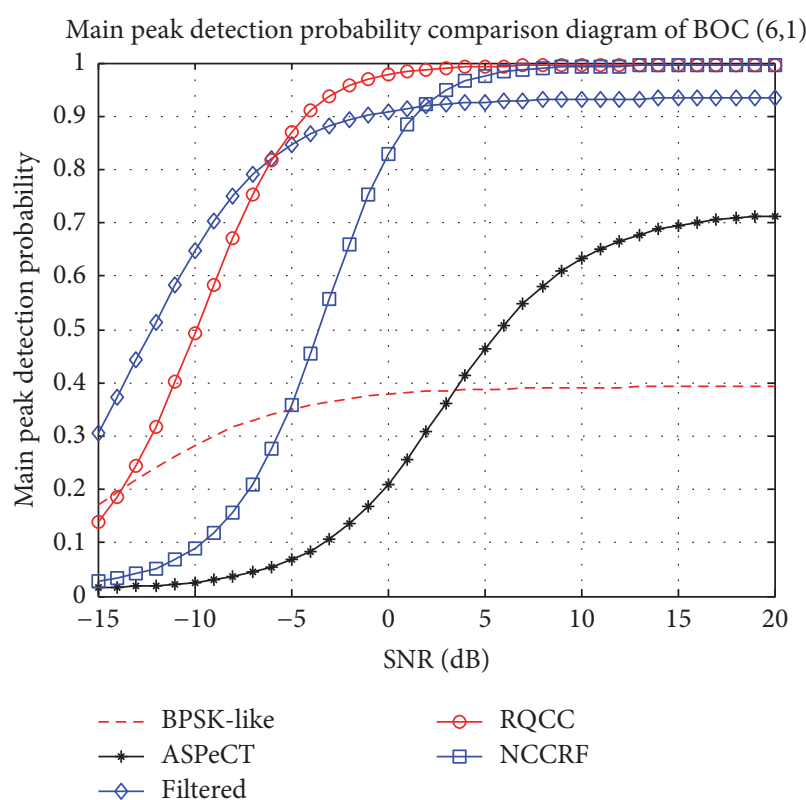

FIGURE 15: The MPDR comparison of $\operatorname{BOC}(6,1)$.

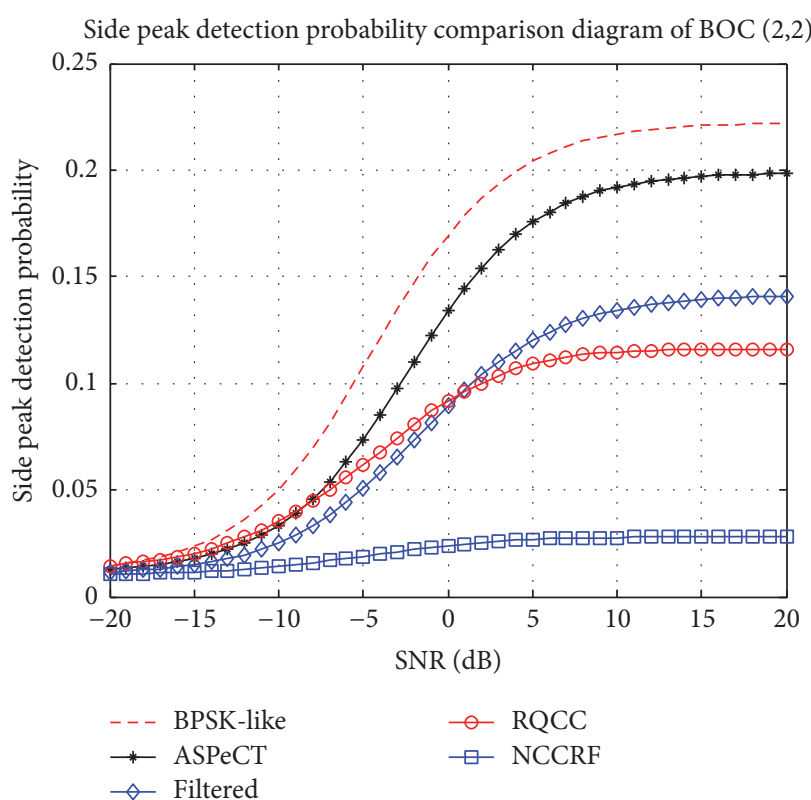

FIGURE 16: The SPDR comparison of $\operatorname{BOC}(2,2)$.

high. In general, the new algorithm has better first side peaks restrained performance in different modulation coefficient for all BOC signals.

\section{Conclusions}

On the basis of analyzing the limitations for the existing correlation reconstruction methods, a method of NCCFR is been proposed. In order to verify the acquisition performance for the new algorithm, the simulation comparison has been analyzed by using different acquisition methods in different 
Side peak detection probability comparison diagram of BOC $(8,4)$

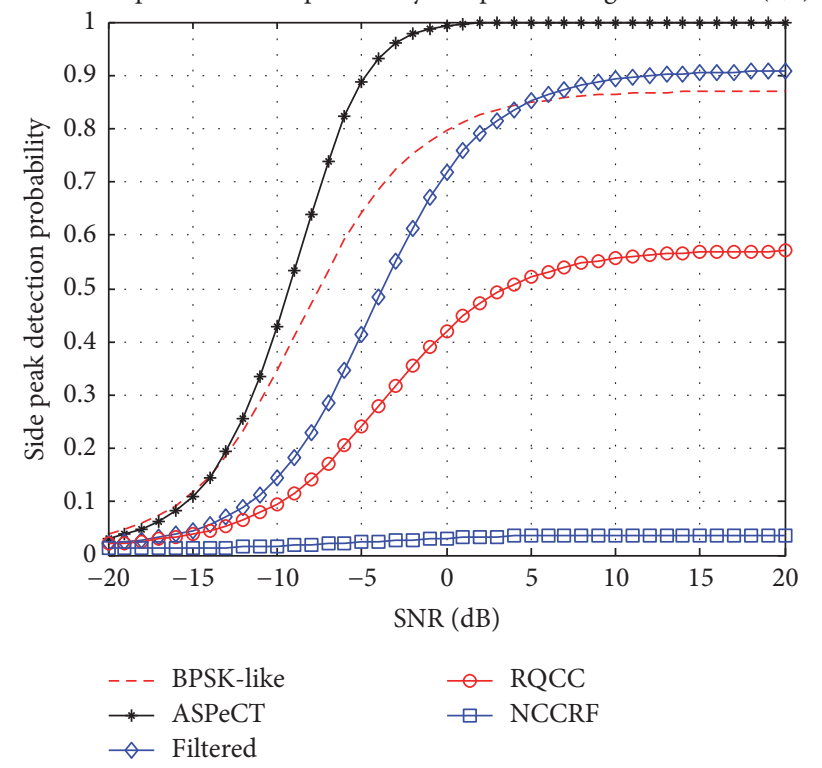

FIgURE 17: The SPDR comparison of $\operatorname{BOC}(8,4)$.

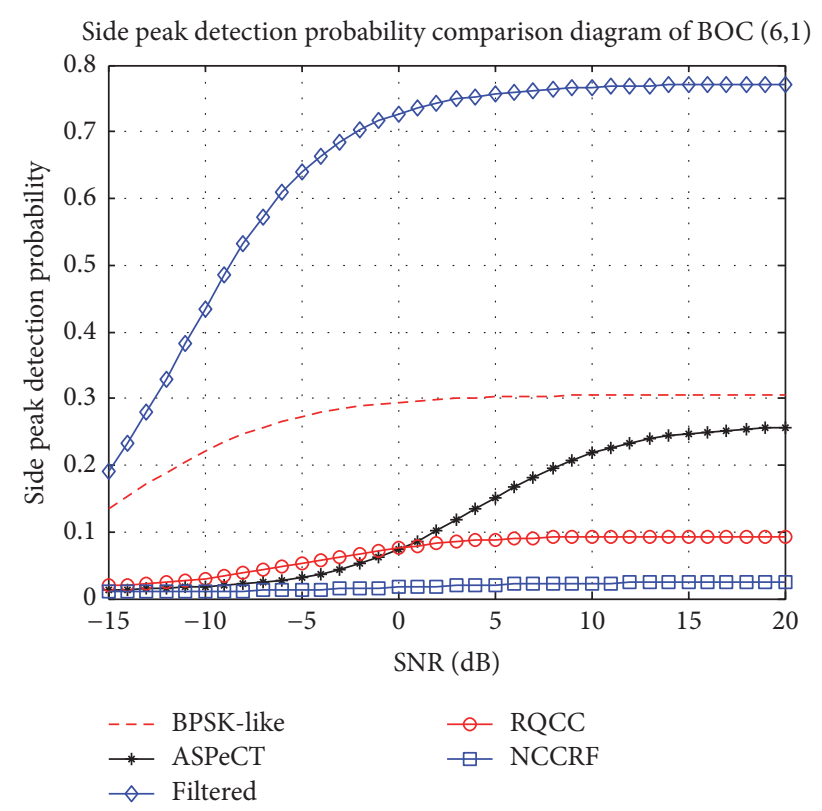

FIGURE 18: The SPDR comparison of $\operatorname{BOC}(6,1)$.

SNR conditions based on BOC $(2,2)$, BOC $(8,4)$, and BOC $(6,1)$. The simulation results show that the proposed algorithm is better than all of the other algorithms in enhancing the main peak and restraining the side peaks, when the modulation coefficients are of low order. And the results show that the new algorithm has better performance than other algorithms when the modulation coefficients are of high order.

\section{Conflicts of Interest}

The authors declare that they have no conflicts of interest.

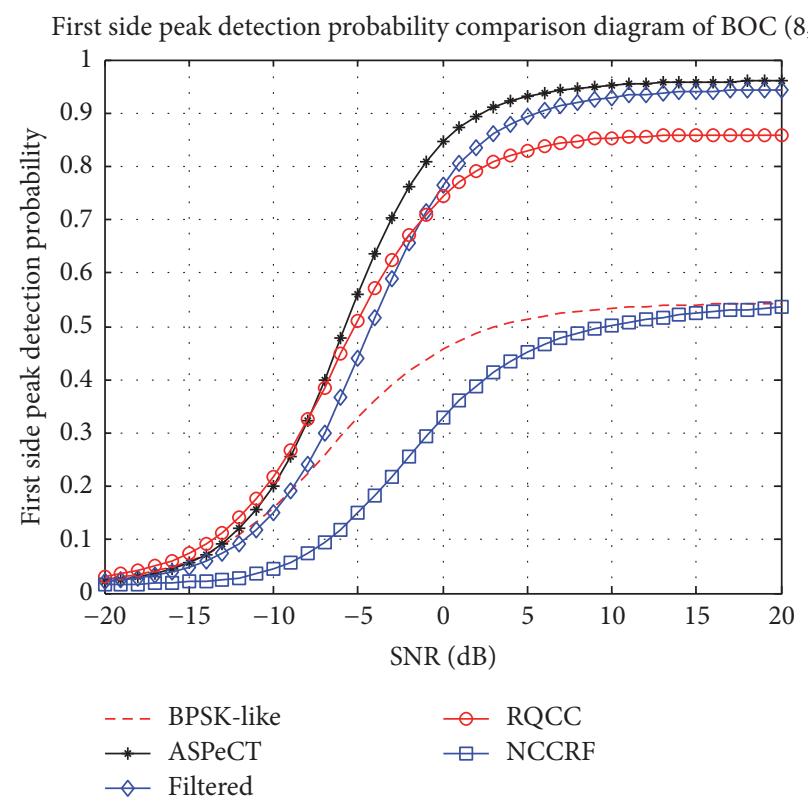

FIGURE 19: The FSPDR comparison of $\operatorname{BOC}(8,4)$.

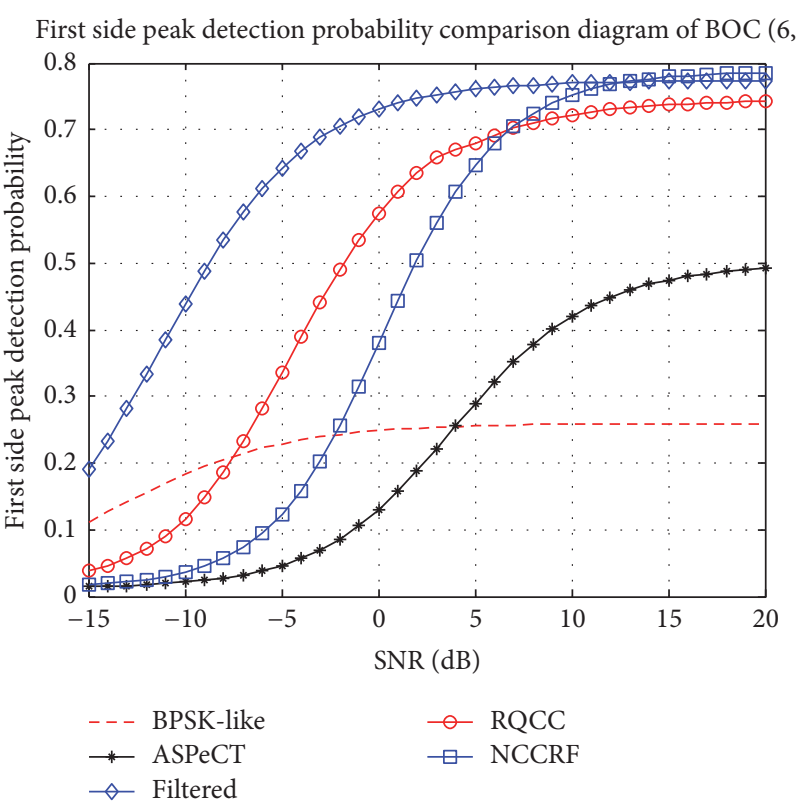

Figure 20: The FblackSPDR comparison of $\operatorname{BOC}(6,1)$.

\section{Acknowledgments}

This work was supported by the National Natural Science Foundation of China (no. 61501309), the Program for Liaoning Innovative Research Team in University (no. LT2011005), New Century Program for Excellent Talents of Ministry of Education of China (no. NCET-11-1013), and the China Postdoctoral Science Foundation (no. 2015M580231; no. 2017T100185). 


\section{References}

[1] P. Li, F. Gao, and Q. Li, "An improved unambiguous acquisition scheme for BOC $(\mathrm{n}, \mathrm{n})$ signals," in Proceedings of the International Conference on Wireless Communications Signal Processing WCSP '15, pp. 1-6, 2015.

[2] S. Yoon, S. C. Kim, J. Heo, I. Song, and S. Y. Kim, “Twin-cell detection (TCD): a code acquisition scheme in the presence of fractional doppler frequency offset," IEEE Transactions on Vehicular Technology, vol. 58, no. 4, pp. 1797-1803, 2009.

[3] D. Chong, Y. Lee, I. Song, and S. Yoon, "A two-stage acquisition cheme based on multiple correlator outputs for UWB signals," IEICE Electronics Express, vol. 8, no. 7, pp. 436-442, 2011.

[4] Y. Lee, D. Chong, I. Song, S. Y. Kim, G.-I. Jee, and S. Yoon, "Cancellation of correlation side-peaks for unambiguous BOC signal tracking," IEEE Communications Letters, vol. 16, no. 5, pp. 569-572, 2012.

[5] H. Chen, W. Jia, and M. Yao, "Cross-correlation function based multipath mitigation technique for cosine-BOC signals," Journal of Systems Engineering and Electronics, vol. 24, no. 5, Article ID 00087, pp. 742-748, 2013.

[6] W.-L. Mao, C.-S. Hwang, C.-W. Hung, J. Sheen, and P.-H. Chen, "Unambiguous BPSK-like CSC method for Galileo acquisition," in Proceedings of the 18th International Conference on Methods and Models in Automation and Robotics (MMAR '13), pp. 627632, IEEE, Międzyzdroje, Poland, August 2013.

[7] A. Burian, E. S. Lohan, and M. Renfors, "BPSK-like methods for hybrid-search acquisition of galileo signals," in Proceedings of the IEEE International Conference on Communications (ICC '06), pp. 5211-5216, IEEE, Istanbul, Turkey, July 2006.

[8] O. Julien, C. Macabiau, M. E. Cannon, and G. Lachapelle, "ASPeCT: unambiguous sine-BOC $(\mathrm{n}, \mathrm{n})$ acquisition/tracking technique for navigation applications," IEEE Transactions on Aerospace and Electronic Systems, vol. 43, no. 1, pp. 150-162, 2007.

[9] E. F. Brickell, D. M. Gordon, K. S. Mccurley et al., "Fast exponentiation with precomputation," in Proceedings of the Eurocrypt on Advances in Cryptology, vol. 658, pp. 200-207, Springer-Verlag, New York, NY, USA, 1993.

[10] L. Yanzan, The Fast Acquisition Technology of Multi-Mode Navigation Signals Based on The BOC Signals, Dalian University, Dalian, China, 2013.

[11] W. Cui, D. Zhao, J. Liu, S. Wu, and J. Ding, "A novel unambiguous acquisition algorithm for BOC $(\mathrm{m}, \mathrm{n})$ signals," in Proceedings of the 5th IEEE International Conference on Signal Processing, Communications and Computing, ICSPCC '15, IEEE, Ningbo, China, September 2015.

[12] Y. Zhang, W. Luy, and D. Yu, "A fast acquisition algorithm based on FFT for BOC modulated signals," in Proceedings of the 35th IEEE Region 10 Conference, TENCON '15, IEEE, Macao, China, November 2015.

[13] Z. Yang, Z. Huang, and S. Geng, "Unambiguous acquisition performance analysis of $\mathrm{BOC}(\mathrm{m}, \mathrm{n})$ signal," in Proceedings of the International Conference on Information Engineering and Computer Science, ICIECS '09, IEEE, Wuhan, China, December 2009.

[14] F. Liu, Y.-X. Feng, and M.-H. Tian, "The main peak estimate algorithm based on $\mathrm{BOC}(2 \mathrm{n}, \mathrm{n})$ signal," in Proceedings of the $3 \mathrm{rd}$ IEEE International Conference on Advanced Computer Control, ICACC '11, pp. 165-168, IEEE, Harbin, China, January 2011.

[15] O. M. Mubarak, "Performance comparison of multipath detection using early late phase in BPSK and BOC modulated signals," in Proceedings of the 7th International Conference on Signal Processing and Communication Systems, ICSPCS '13, IEEE, Carrara, VIC, Australia, December 2013.

[16] L. Yang, C.-S. Pan, Y.-X. Feng, and Y.-M. Bo, "A new algorithm for synchronous main lobe detection for BOC modulated navigation signals," Journal of Astronautics, vol. 31, no. 8, pp. 2008-2014, 2010.

[17] A. Wang, J. Wang, and B. Xue, "Acquisition of BOC $(n, n)$ with large Doppler," in Proceedings of the 7th International Conference on Wireless Communications, Networking and Mobile Computing, WiCOM '11, IEEE, Wuhan, China, September 2011.

[18] F. Shen, G. Xu, and D. Xu, "Unambiguous acquisition technique for cosine-phased binary offset carrier signal," IEEE Communications Letters, vol. 18, no. 10, pp. 1751-1754, 2014.

[19] Y. Chuanxi, Performance Analysis of Ranging Codes And Study on Acquisition Algorithm of BOC Signal, Chinese Academy of Science (National Time Service Center), Beijing, China, 2013. 


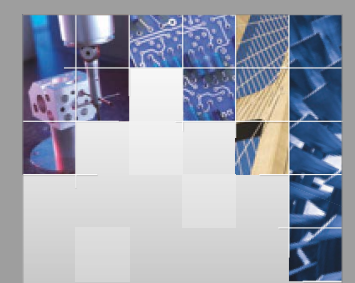

\section{Enfincering}
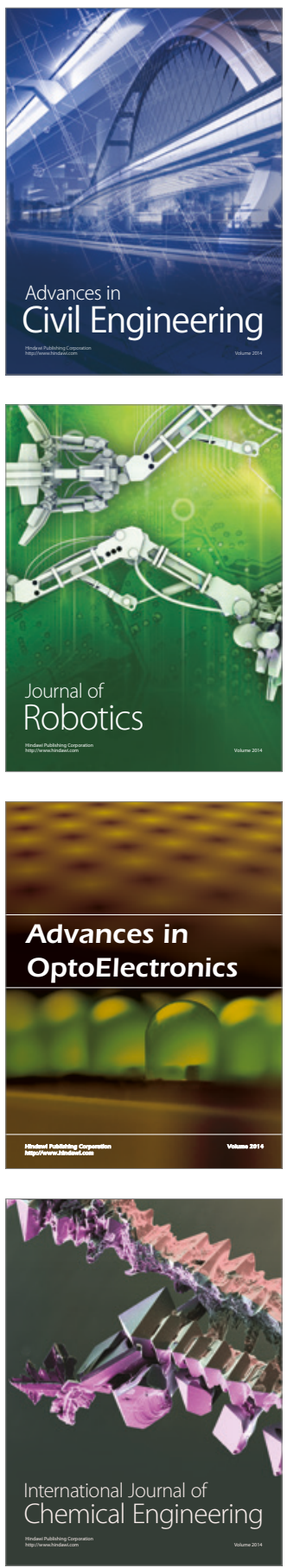

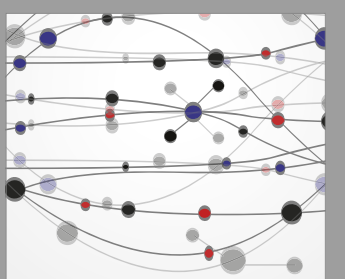

The Scientific World Journal

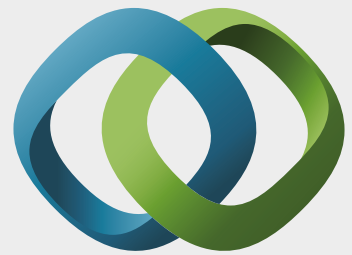

\section{Hindawi}

Submit your manuscripts at

https://www.hindawi.com
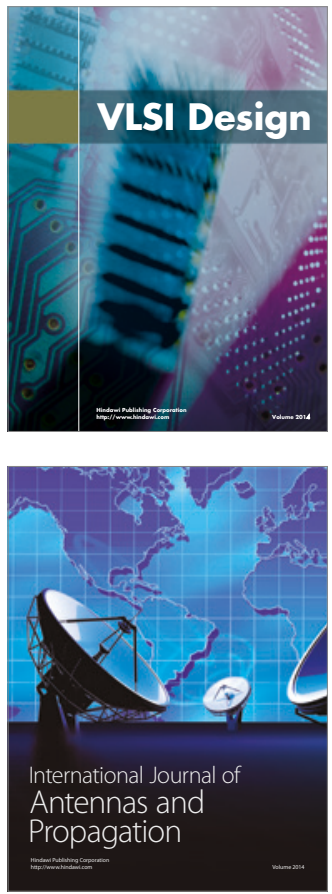

\section{Rotating}

Machinery
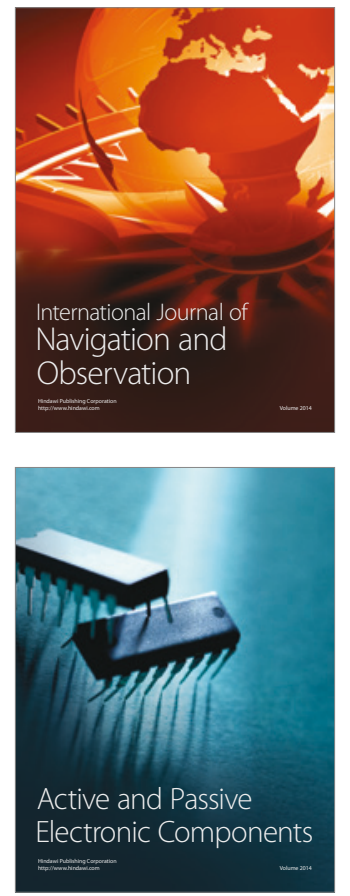
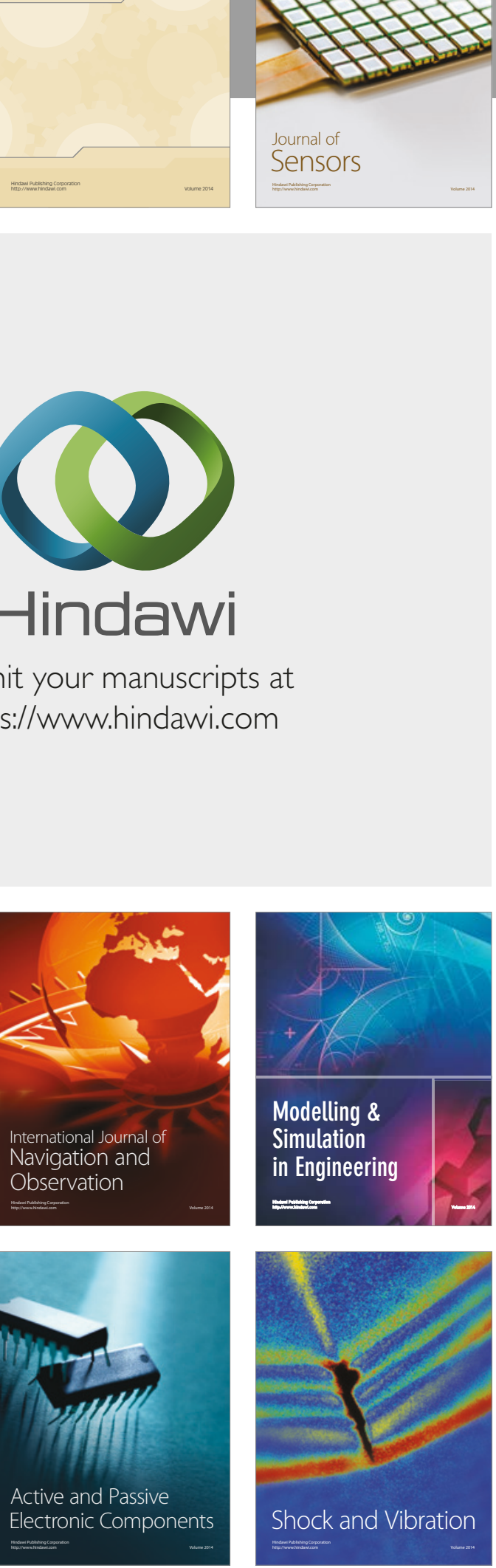
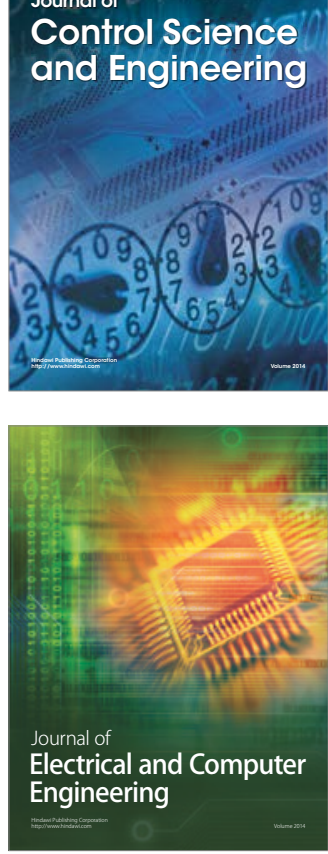

Distributed

Journal of

Control Science

and Engineering
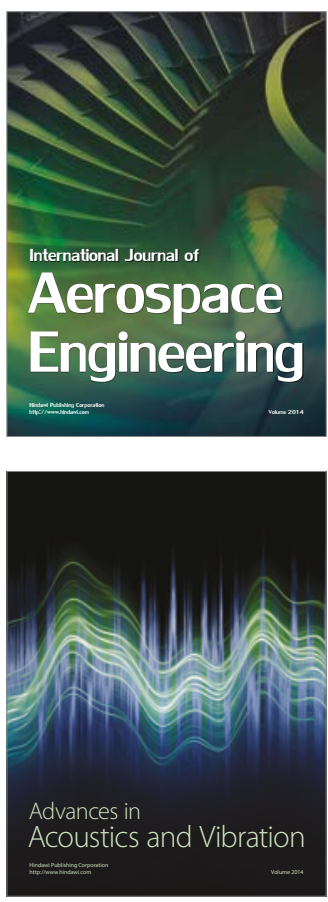

Sensor Networks 